\title{
Maternal Anemia and Iron Deficiency Anemia: Similarities and Singularities
}

Figueiredo ACMG ${ }^{1 *}$, Gomes-Filho IS ${ }^{2}$, Silva RB' ${ }^{1}$, Cruz SSD ${ }^{3}$ and Pereira MG ${ }^{1}$

${ }^{1}$ University of Brasilia, Distrito Federal, Brazil

${ }^{2}$ Department of Health, Feira de Santana State University, Brazil

${ }^{3}$ Department of Epidemiology, Federal University of Recôncavo da Bahia

\begin{abstract}
Maternal anemia is a major global public health problem, and although widely discussed, there are few studies investigating the condition in pregnant women. In this article, issues related to the diagnosis, biological mechanism and prevalence of maternal anemia. In addition, iron deficiency anemia will be considered a proxy for maternal anemia. In previous studies, the concepts of maternal anemia have been controversial. It is also noted that isolated actions are not sufficient to combat this disease, and policies to address the primary causes of the associated nutritional deficiencies are necessary.
\end{abstract}

Keywords: Anemia; Iron deficiency; Iron deficiency anemia; Pregnancy outcome; Differential diagnosis

\section{Introduction}

Anemia is a relevant problem worldwide, typical of large urban centers and affecting countries of various economic levels from North America to Sub-Saharan Africa [1]. On a biological level, this deficiency is characterized by a reduction in hemoglobin levels due to a lack of essential nutrients, such as B vitamins, zinc, protein and iron $[1,2]$. Both the definition and the classification of anemia were determined by consensus by multiple international institutions approximately two decades ago at the New York Summit Meeting [2]. As a result of this discussion, anemia has become a priority for many countries, with the main goal being its reduction across different population groups [3]. There are many studies with different methodological strategies focusing on anemia [4-12]. Such studies include distinct population subgroups and different sample sizes, producing conflicting results [12]. Furthermore, the majority of studies use hemoglobin level as the only diagnostic criterion, ignoring the various types of anemia [12].

The World Health Organization (WHO) considers iron deficiency anemia to be the main cause of maternal anemia [1]. While some studies consider hemoglobin level the only diagnostic criterion for iron deficiency anemia, other studies show that the frequency of iron deficiency is lower when diagnosed using both hemoglobin and ferritin levels [5-7,13-16]. Therefore, there is no consensus that maternal anemia has a frequency similar to that of iron deficiency anemia Thus, we aimed to review the main aspects of health-disease-care process of maternal anemia, focusing on the comparison of maternal and iron deficiency anemia. Biological mechanisms, diagnostic criteria and prevalence were discussed [5-7,13-17].

\section{Materials and Methods}

\section{Eligibility criteria}

Cross sectional and baseline of cohort studies assessing the maternal anemia or iron deficiency anemia were considered for inclusion. Publication date or languages were not exclusion criteria.

\section{Information sources}

We searched for eligible articles on December $17^{\text {th }}, 2017$, on the following electronic databases: Medline via PubMed, EMBASE, Scopus, Web of Science, SciELO and Lilacs. We also gray literature and hand searched reference lists from selected articles.

\section{Search strategies}

Mesh and similar terms were combined using Boolean operators (AND, OR), and the search strategy below was primarily used for PubMed and afterwards adapted for the remaining databases: (anemia OR anaemia OR anemia, iron deficiency) AND (Pregnancy OR Pregnant Women OR Gravidity OR Maternal exposure OR Mother OR Gestation) AND (Prevalence OR Frequency) AND (Cross Sectional Studies OR Cross Sectional Study Cohort studies OR Longitudinal Studies OR FollowUp Studies OR Prospective studies OR Cohort OR Longitudinal OR Prospective OR Retrospective OR Incidence study OR Follow up).

\section{Studies selection}

After duplicate removal, titles and abstracts were screened for eligible articles independently by two reviewers (ACMGF and SSC). Two reviewers selected articles by reading titles and abstracts. During the process of selecting the articles the researchers were not aware of the decisions made by their colleague. After that, full text articles were assessed for eligibility. Discordant events were resolved by consensus.

\section{Data extraction}

Data extraction was performed independently by two researchars (ACMGF and RBS) and discordant events resolved by consensus. We used a standardized electronic spreadsheet to extract the following information: author's name, year of publication, year of data collection and geographic region, study design, sample size, anemia diagnosis criteria, frequency of maternal anemia and iron deficiency anemia. Authors were contacted for additional information considered important for this review.

\section{Data synthesis}

Information regarding maternal anemia and iron deficiency anemia was descripted and summarized in figures.

*Corresponding author: Ana Claudia Morais Godoy Figueiredo, University of Brasilia Distrito Federal, Brazil, Tel: +55 61 3107-1894; E-mail: aninha_m_godoy@hotmail.com

Received December 22, 2017; Accepted January 05, 2018; Published January 12, 2018

Citation: Figueiredo ACMG, Gomes-Filho IS, Silva RB, Cruz SSD, Pereira MG (2018) Maternal Anemia and Iron Deficiency Anemia: Similarities and Singularities. Health Care Current Reviews 6: 217. doi: 10.4172/2375-4273.1000217

Copyright: (c) 2018 Figueiredo ACMG, et al. This is an open-access article distributed under the terms of the Creative Commons Attribution License, which permits unrestricted use, distribution, and reproduction in any medium, provided the original author and source are credited. 


\section{Results and Discussion}

In the search of the databases, 2243 records were identified. After removing the duplicates and reading titles and abstracts, 192 articles were selected for full reading. Only 36 texts met the eligibility criteria of this review (Figure 1).

\section{Biological mechanisms and diagnostic criteria for anemia in pregnant women}

Basal hemoglobin, which is produced in the bone marrow through erythropoiesis, is a protein formed by four amino acid chains that has one heme peptide in each of the four globins $[17,18]$. The heme group comprises porphyrin and a central iron ion responsible for oxygen transport and energy production inside the cell. Hemoglobin is responsible for red blood cell formation, while hematocrit is the percentage of red blood cell volume $[17,18]$. From a physiological perspective, pregnant women are most vulnerable to anemia due to their increased nutrient needs, pregnancy-related changes and fetal growth $[19,20]$. Anemia is closely related to blood loss and/or decreased hemoglobin, which can occur due to a lack of erythrocyte production or to the hemodilution inherent in pregnancy $[19,20]$.

Hemodilution results from an increase of $50 \%$ in plasmatic volume beginning in the sixth gestational week and continuing to the end of pregnancy to compensate for increased cardiac output and to meet fetal demands $[18,21]$. Hemodilution decreases red blood cells/ hemoglobin in the blood; however, it does not impact the body's total oxygen transport capacity [22]. Notably, the reduction in the number of circulating red blood cells decreases the lifespan of these cells from $18 \%$ to $33 \%$ [23-28]. Thus, red blood cell depletion may lead to a reduction in iron in the blood [29]. A persistent decrease in iron ions can promote iron deficiency anemia because unavailability of this metal prevents the formation of new hemoglobin $[17,18]$. In the event of a decrease in iron levels, the human body has additional sources of this nutrient in the liver. Kupffer cells are responsible for identifying erythrocytes in decay and for stimulating macrophages to remove these senescent cells through phagocytosis [17]. This process will increase iron availability in the intracellular medium that can be exported through ferroportin (FPT) to other cells or stored in the macrophages themselves [17].

There are two sources of dietary iron: 1) animal foods provide heme or ferrous $\left(\mathrm{Fe}^{+2}\right)$ iron and 2) vegetable foods provide non-heme or ferric $\left(\mathrm{Fe}^{+3}\right)$ iron $[17,24]$. Ferrous iron is absorbed into the interior of the cell through heme carrier protein (HCP) [17,24]. Ferric iron is synthesized in enteric cells, duodenal cytochrome b (Dcytb), where it becomes ferrous and is conducted to the intracellular medium through divalent metal transporter protein (DMT-1) [17,24]. Ferroportin transports ferrous iron, which is synthesized in ferritin, to the extracellular medium $(17,24)$. Ferritin conduction occurs after this step through the carrier protein transferrin to the place where hemoglobin is produced [17]. The WHO criteria for maternal anemia area hemoglobin of less than $11.0 \mathrm{~g} / \mathrm{dL}$ or a hematocrit of $33 \%$ or less, regardless of the gestational trimester or type of anemia, which differs from those of previous investigations [1,4,25-28]. Studies indicate that the cut-off for the diagnosis of maternal anemia in the second trimester should be in the range of 10 to $10.5 \mathrm{~g} / \mathrm{dL}$ or two or less standard deviations from the mean of hemoglobin and/or hematocrit $[25,26]$. Iron deficiency anemia is characterized by ferritin levels of less than $15 \mathrm{fL}$ and is related to low levels of hemoglobin $(<11 \mathrm{~g} / \mathrm{dL})[4,29]$. When iron deficiency anemia occurs, serum ferritin is decreased and/or transferrin is increased $[4,29]$. Other types of anemia can be diagnosed during pregnancy, even if blood iron levels are normal, such as anemia of chronic disease, also known as anemia of inflammation [30].

\section{Prevalence of maternal and iron deficiency anemia}

Maternal Anemia reaches across social strata and is considered the most prevalent nutritional deficiency in the world [1] (Figure 2). The WHO classification of the prevalence of maternal anemia is as follows: 1) normal - 0 to $4.9 \%$; 2) mild - 5 to $19.9 \%$; 3 ) moderate -20 to $39.9 \%$; and 4 ) severe $\geq 40 \%$ (Figure 3 ). Notably, there is considerable variability in the prevalence of maternal anemia worldwide, especially when comparing developed countries with economically disadvantaged countries [1]. In 2011, the global frequency of iron deficiency anemia in pregnant women was $19.2 \%$ (95\% CI: 17.1-21.5\%) [5]. The worldwide frequency of the condition was as follows: the Americas and the Caribbean - 15.2\% (95\% CI: 11.7\%; 18.6\%); Europe - 16.2\% (95\% CI: 12.6\%; 19.7\%); Oceania - 17.2\% (95\% CI: 9.7\%; 25.6\%); Asia - 19.8\% (95\% CI: $15.8 \% ; 23.5 \%)$; and Africa - 20.3\% (95\% CI: $18.3 \%$; $23.4 \%)$ [15].

These results were obtained based on the supplementation dosage of ferrous sulfate taken by pregnant women [15]. Some investigations performed on the American continent show the profile of maternal anemia. In Alaska, United States of America, between 1993 and 2006, it was reported that the prevalence of maternal anemia was $18 \%$ [31]. It is estimated that in Mexico in 2012, 21\% of pregnant women were diagnosed with anemia and that only half of cases were caused by iron

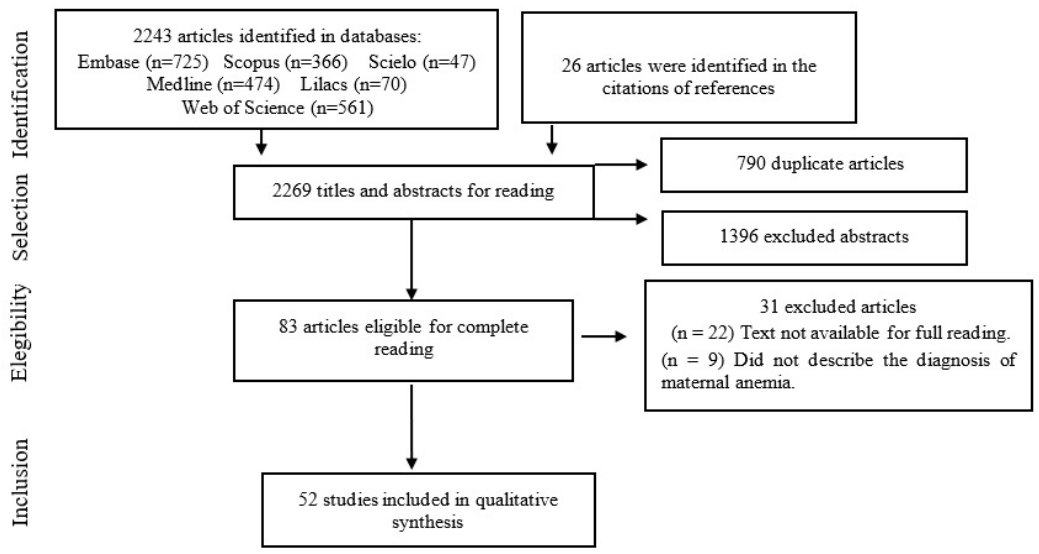

Figure 1: Flowchart of the search, selection and inclusion of the studies. 


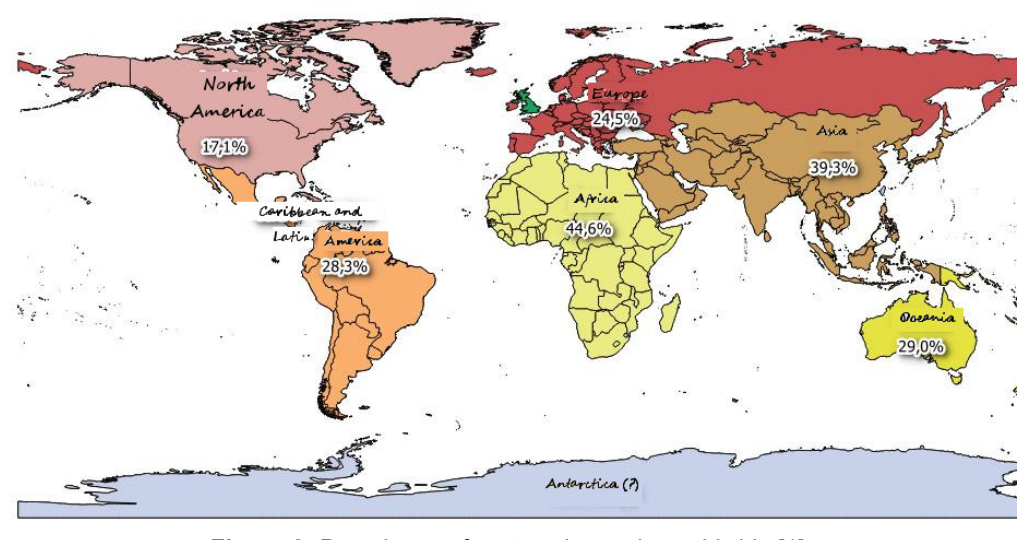

Figure 2: Prevalence of maternal anemia worldwide [1].

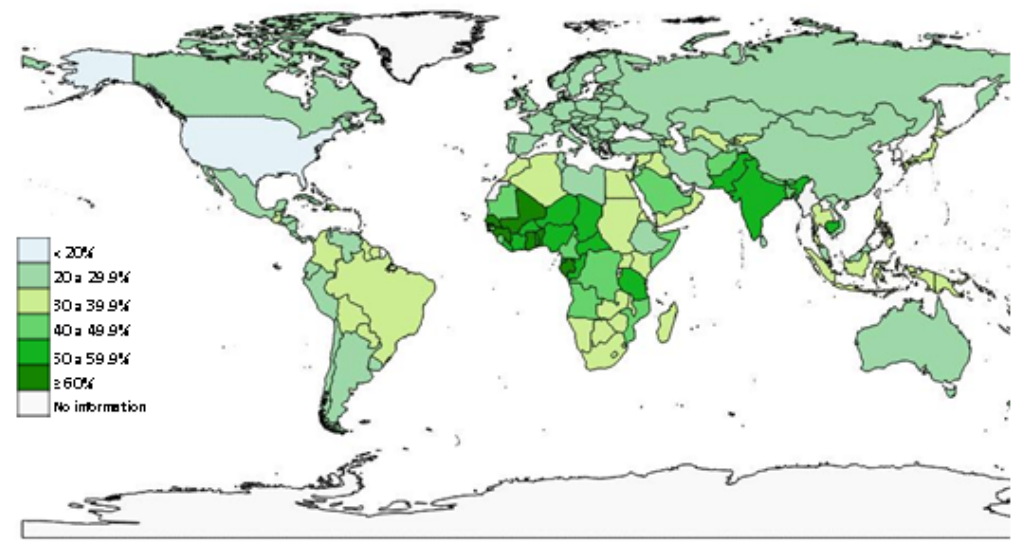

Figure 3: Prevalence of maternal anemia worldwide according to occurrence levels [1].

deficiency [32]. In Cuba and Peru, 2011, the prevalence of maternal anemia was $29.7 \%$ and $28 \%$, respectively [33,34]. A study performed in Brazil between 2000 and 2001 [4] showed that $56 \%$ of pregnant women enrolled in the study had a diagnosis of maternal anemia and that approximately $11 \%$ of those women had iron deficiency anemia [4]. The average frequency of maternal anemia in European countries is $24.5 \%$ [1]. In Switzerland, the occurrence of iron deficiency (32.2\%) and anemia related to iron deficiency (6.5\%) differed [7]. It should be emphasized that other types of anemia were found in $11.8 \%$ of pregnant women in this longitudinal investigation [7]. Data from a cohort study of 1,478 pregnant women showed that $15.8 \%$ of French women had maternal anemia in 2013 and 2014 [10]. Of these women, approximately $31 \%$ had iron deficiency [10]. Asia has the second-highest rate of maternal anemia in the world [1]. In a prospective investigation performed in India, a country considered to have severe maternal anemia, the rate varied between $41 \%$ and $55 \%$ in the first and third trimesters of gestation, respectively. However, the prevalence of iron deficiency anemia was $3.6 \%$ and $5.6 \%$, respectively [16]. In Japan, the frequency of maternal anemia in 2011 increased substantially between the first and second trimesters, (4.5\% and $44.1 \%$, respectively) [11].

In Nigeria, a Sub-Saharan country, the percentage of women with maternal anemia in 2014 was approximately $59 \%$ at the end of gestation [35]. In Algeria, the occurrence of maternal anemia was approximately $47 \%$ in 2010 [36]. No studies reporting the frequency of iron deficiency and iron deficiency anemia in pregnant women in African countries were found. Research performed in Oceania showed no consensus regarding maternal anemia indicators [37-39]. Betwen 1999 and 2005, the occurrence of maternal anemia in southern Australia was 7.1\% [37]. In New Zealand, 2013 data indicate a frequency of maternal anemia of $54.5 \%$; however, only $6.3 \%$ and $5.8 \%$ of the pregnant women had iron deficiency and iron deficiency anemia, respectively [39]. Another relevant indicator for this hematological disorder concerns the severity of maternal anemia [1].

The data show that the frequencies of mild and moderate maternal anemia are elevated among pregnant women [14,34,36,4046] and that severity levels vary greatly across countries (Figure 4). From the perspective of minimizing the occurrence of maternal anemia, prophylactic actions, such as ferrous sulfate and folic acid supplementation, have been recommended [28,47]. However, between 1995 and 2011, worldwide epidemiological indicators of maternal anemia decreased by only $3.6 \%$ (from $41.8 \%$ to $38.2 \%$ ) [1]. The use of these supplements has not been sufficient to markedly reduce the prevalence of maternal anemia worldwide in recent years [2]. The data we presented indicate discrepancies in the prevalence of maternal anemia in different countries. In the majority of studies, estimates of iron deficiency anemia are always lower than those for maternal anemia [5,13-15]. However, official WHO documents indicate that these events generally occur with similar frequencies [1]. 


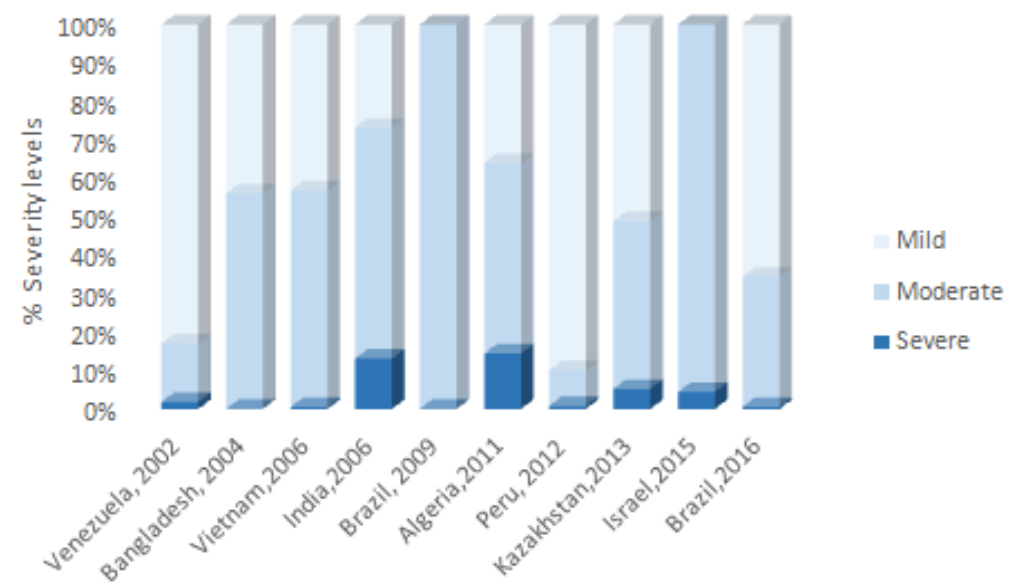

Figure 4: Diagnosis of maternal anemia in different countries by severity, with their respective percentages $[14,39,41,44,48-52]$

\section{Is iron deficiency anemia a proxy for maternal anemia?}

The WHO definition of anemia in pregnant women is a condition caused by cumulative iron deficiency [48]. Therefore, the question is whether maternal anemia is a proxy for iron deficiency anemia [2]. However, there is no consensus regarding this statement $[5,7,13,15,16]$. Various studies consider iron deficiency an essential condition in the occurrence of maternal anemia, as those studies concluded that most women have iron deficiency during gestation [49]. It is estimated that iron deficiency is 2.5 times more frequent than anemia in pregnant women; therefore, when a pregnant woman has low hemoglobin, it is mistakenly characterized as maternal anemia rather than iron deficiency anemia [49]. The WHO supports the argument that hemoglobin levels and/or hematocrit are good markers of anemia; these markers offer cost-effective ways of screening for the disease and, that in the absence of laboratory tests that allow for differential diagnosis, the women would be diagnosed with iron deficiency anemia $[1,48]$. The statement is based on the fact countries have financial resources that are too limited to use more than one test to diagnose anemia in pregnant women, this would entail a health cost of approximately $50 \%$ more than necessary $[4,43]$. Studies comparing levels of hemoglobin with ferritin (the gold standard) find that these results are quite close [5,13-15]. Nonetheless, the use of hemoglobin levels as the only test for maternal anemia is not ideal, as it cannot accurately diagnose the type of anemia because of its low sensitivity $[4,21]$. The use of at least two criteria to clarify the etiology of anemia might improve the specificity of the test and reduce the probability of false positives; that is, the possibility of diagnosing iron deficiency anemia in women who do not have iron deficiency [4,21]. The appropriate use of hematological markers could promote investigations into the type of anemia [48]. For example, the number of red cells might help in the classification of anemia and direct health professionals to request complementary tests, such as ferritin levels $[48,49]$. Red blood cell values above 4 million and hemoglobin levels below $11 \mathrm{~g} / \mathrm{dL}$ are suggestive of iron deficiency anemia, requiring confirmation by the level of ferritin (<15 fL) for diagnosis [49-51].

The absence of a differential diagnosis or the inability of health professionals to characterize the type of anemia, as well as unawareness of its magnitude, may be an impediment to adequate therapy at the population level. That is, iron supplementation might be erroneously recommended by health professionals who are not certain what type of anemia is to be treated in pregnant women $[47,52]$. Many authors dis- agree that maternal anemia might be representative of iron deficiency anemia because some studies show discrepancies in the prevalence of both types of anemia $[4,6,7,16,21,43]$. Such research shows that the occurrence of iron deficiency anemia is, in most investigations, expressly lower than that of maternal anemia $[4,6,7,16,21,43]$. There has been an increase in the number of publications regarding diagnostic criteria for iron deficiency anemia in the last decade $[4,5,21]$. Studies that use serum ferritin and/or transferrin levels to diagnose iron deficiency anemia report results that are more consistent for the population of pregnant women $[4,6,7,16,21,52]$ than studies that used only hemoglobin levels [5,13-15]. The WHO maternal anemia criteria are considered inappropriate by some authors, when there is the intention to estimate iron deficiency anemia $[2,4,6,7,21]$. This issue is related to the disease pathophysiology, given that the origin of maternal anemia might not be iron deficiency but other uninvestigated causes. The premise that there is a resemblance between maternal anemia and iron deficiency anemia has driven the indiscriminate supplementation of ferrous sulfate during pregnancy. For this reason, the current literature, contrary to the idea that iron deficiency anemia is a proxy for maternal anemia, emphasizes that the use of specific and sensitive diagnostic criteria is very important to minimize adverse effects that could be caused by inadequate supplementation $[4,21]$.

\section{Conclusion}

The majority of studies included in this review did not perform anemia differential diagnoses during pregnancy. The absence of anemia differential diagnoses might confound the magnitude of the problem. In other words, the causal mechanisms for both maternal and iron deficiency anemia may differ. as it can be related to other factors rather than iron deficiency, such as under nutrition, infection or other events prior to pregnancy. Preventive actions are of major importance to minimize the occurrence of maternal anemia, as is the accurate diagnosis of the disease, since isolated actions alone are insufficient to impact the nutritional status of the population.

\section{Conflicts Interests}

The authors declare that they have no conflicts of interests.

\section{References}

1. World Health Organization (2015) The global prevalence of anaemia in 2011 Geneva: World Health Organization. 
2. Batista-Filho M, Souza Al, Bresani CC (2008) Anemia as a public health problem: The current situation. Ciênc. saúde coletiva 13: 1917-1922.

3. UNICEF (1990) Strategy for improved nutrition of children and women in developing countries. New York: UNICEF 38

4. Bresani CC, Souza Al, Batista-Filho M (2007) Anemia and iron deficiency in pregnant women: Disagreements among the results of a cross-sectional study. Rev Bras Saúde Matern Infant 7: S15-S21

5. Banjari I, Kenjerić D, Mandić ML (2015) What is the real public health significance of iron deficiency and iron deficiency anemia in Croatia? A population-based observational study on pregnant women at early pregnancy from eastern Croatia. Cent Eur J Public Health 23: 98-103.

6. Vandevijvere S, Amsalkhir S, Van Oyen H (2013) Iron status and its determinants in a nationally representative sample of pregnant women. J Acad Nutr Diet 113: 659-666

7. Bencaiova G, Breymann C (2014) Mild anemia and pregnancy outcome in a Swiss collective. J Pregnancy 2014: 1-7

8. Barroso F, Allard S, Kahan BC (2011) Prevalence of maternal anaemia and its predictors: A multi-centre study. Eur J Obstet Gynecol Reprod Biol 159: 99-105.

9. Eisele L, Dürig J, Broecker-Preuss M (2013) Prevalence and incidence of anemia in the German Heinz Nixdorf Recall Study. Ann Hematol 92: 731-737.

10. Harvey T, Zkik A, Auges M (2016) Assessment of iron deficiency and anemia in pregnant women: an observational French study. Womens Health (Lond). 12: $95-102$.

11. Jwa SC, Fujiwara T, Yamanobe $Y$ (2015) Changes in maternal hemoglobin during pregnancy and birth outcomes. BMC Pregnancy Childbirth 15: 80

12. Lopez A, Cacoub P, Macdougall IC (2016) Iron deficiency anaemia. Lancet 387 907-916

13. Casanova BF, Sammel MD, Macones GA (2005) Development of a clinical prediction rule for iron deficiency anemia in pregnancy. Am J Obstet Gynecol 193:460-466

14. Aikawa R, Ngyen CK, Sasaki S (2006) Risk factors for iron-deficiency anaemia among pregnant women living in rural Vietnam. Public Health Nutr 9: 443-448.

15. Black RE, Victora CG, Walker SP (2013) Maternal and child under nutrition and overweight in low-income and middle-income countries. Lancet 82: 427-451.

16. Menon KC, Ferguson EL, Thomson CD (2016) Effects of anemia at different stages of gestation on infant outcomes. Nutrition 32: 61-65.

17. Donovan A, Roy C, Andrews N (2006) The ins and outs of iron homeostasis. Physiology 21: 115-23.

18. Taylor DJ, Mallen C, McDougall N (1982) Effect of iron supplementation on serum ferritin levels during and after pregnancy. J Obstet Ginecol 89: 1011-1017.

19. Tapia ME (2010) Prevalência de anemia nas gestantes atendidas no Sistema Unico de Saúde-Secretaria Municipal de Saúde-Prefeitura de Belo Horizonte. RBAC 42: 277-281.

20. Souza Al, Batista-Filho M (2003) Dianosis and treatment of nutritional anemia in pregnancy: Consensus and controversies. Rev Bras Saúde Matern Infant 3:473-479.

21. Bresani CC, Braga MC, Felisberto DF (2013) Accuracy of erythrogram and serum ferritin for the maternal anemia diagnosis (AMA): A phase 3 diagnostic study on prediction of the therapeutic responsiveness to oral iron in pregnancy. BMC Pregnancy Childbirth 13: 13.

22. World Health Organization (2003) The clinical use of blood in medicine obstetrics, paediatrics surgery and anaesthesia and trauma and burns. Geneva: World Health Organization, p: 344.

23. Cançado RD, Chiattone CS (2002) Anemia of chronic disease. Rev Bras de Hematol e Hemot 24: 127-136.

24. Hoffbrand A, Pettit F, Moss $P$ (2006) Essential haematology

25. Milman N, Byg KE, Agger AO (2000) Hemoglobin and erythrocyte indices during normal pregnancy and postpartum in 206 women with and without iron supplementation. Acta Obstet Gynecol Scand 79: 89-98.

26. Yip R (2000) Significance of an abnormally low or high hemoglobin concentration during pregnancy: Special consideration of iron nutrition. Am J Clin Nutr 72: 272S-9S
27. Centers for Disease Control and Prevention (CDC) (1989) CDC criteria for anemia in children and childbearing-aged women. MMWR Morb Mortal Wkly Rep 38: 400-404.

28. Centers for Disease Control and Prevention (1998) Recommendations to prevent and control iron deficiency in the United States Centers for Disease Control and Prevention. MMWR Recomm Rep 47: 1-36.

29. Peña-Rosas JP, De-Regil LM, Dowswell T (2012) Daily oral iron supplementation during pregnancy. Cochrane Database Syst Rev 12: CD004736.

30. Weiss G, Goodnough LT (2005) Anemia of chronic disease. N Engl J Med 352 1011-1023.

31. Gessner BD (2009) Geographic and racial patterns of anemia prevalence among low-income Alaskan children and pregnant or postpartum women limit potential etiologies. J Pediatr Gastroenterol Nutr 48: 475-481.

32. Montoya-Romero Jde J, Castelazo Morales E, Valerio Castro E, Velázquez Cornejo G, Nava Muñoz DA, et al. (2012) Review by expert group in the diagnosis and treatment of anemia in pregnant women. Federación Mexicana de Colegios de Obstetricia y Ginecología. Ginecol Obstet Mex 80 9: 563-580.

33. Pita GM, Jiménez S, Basabe B (2014) Anemia in children under five years old in Eastern Cuba, 2005-2011. MEDICC Rev 16: 16-23.

34. Munares-García O, Gómez-Guizado G, Barboza-Del Carpio J (2012) Hemoglobin levels in pregnant women seen in health care centers of the Peruvian Ministry of Health, 2011. Rev Peru Med Exp Salud Publica 29: 329336.

35. Adanikin Al, Awoleke JO, Olofinbiyi BA (2015) Routine iron supplementation and anaemia by third trimester in a Nigerian hospital. Ethiop J Health Sci 25 305-312.

36. Demmouche A, Lazrag A, Moulessehoul S (2011) Prevalence of anaemia in pregnant women during the last trimester: Consequence for birth weight. Eur Rev Med Pharmacol Sci 15: 436-45.

37. Smithers LG, Gialamas A, Scheil W (2014) Anaemia of pregnancy, perinata outcomes and children's developmental vulnerability: A whole-of-population study. Paediatr Perinat Epidemiol 28: 381-90.

38. Tuinakelo LR, Tayler-Smith K, Khogali M (2013) Prevalence of anaemia, syphilis and hepatitis B in pregnant women in Nausori, Fiji. Public Health Action 3: $72-75$.

39. Calje E, Skinner J (2017) The challenge of defining and treating anemia and iron deficiency in pregnancy: A study of New Zealand midwives' management of iron status in pregnancy and the postpartum period. Birth 44: 181-90.

40. Martí-Carvajal A, Peña-Martí G, Comunian G, Muñoz SE (2002) Prevalence of anemia during pregnancy: results of Valencia (Venezuela) anemia during pregnancy study. Arch Latinoam Nutr 52: 5-11.

41. Hyder SM, Persson LA, Chowdhury M (2004) Anaemia and iron deficiency during pregnancy in rural Bangladesh. Public Health Nutr 7: 1065-1070.

42. Toteja GS, Singh P, Dhillon BS (2006) Prevalence of anemia among pregnant women and adolescent girls in 16 districts of India. Food Nutr Bull 27: 311-315

43. Treister-Goltzman Y, Peleg R, Biderman A (2015) Anemia among Muslim Bedouin and Jewish women of childbearing age in southern Israel. Ann Hematol 94: 1777-1784.

44. Pinho-Pompeu M, Surita FG, Pastore DA (2017) Anemia in pregnant adolescents: impact of treatment on perinatal outcomes. J Matern Fetal Neonatal Med 30: 1158-62.

45. Massucheti LM, Corso ACTC, Moreira EAM (2009) Prevalence of anemia in pregnant women assisted in public health services in Florianópolis municipalty (SC). Cad Saúde Colet 10: 427-432.

46. Smagulova IE, Sharmanov TSh, Balgimekov SA (2013) The prevalence of anemia among children and women of reproductive age in Kazakhstan and basis of its prevention. Vopr Pitan 82: 58-63

47. World Health Organization (2016) World Health Organization recommendations on antenatal care for a positive pregnancy experience. Geneva: World Health Organization.

48. Qi L, Van Dam RM, Rexrode K (2007) Heme iron from diet as a risk facto for coronary heart disease in women with type 2 diabetes. Diabetes Care 30 101-106. 
Citation: Figueiredo ACMG, Gomes-Filho IS, Silva RB, Cruz SSD, Pereira MG (2018) Maternal Anemia and Iron Deficiency Anemia: Similarities and Singularities. Health Care Current Reviews 6: 217. doi: 10.4172/2375-4273.1000217

Page 6 of 6

49. Matos JF, Dusse LMSA, Gomes KBG (2012) Blood tests in microcytic and hypochromic anemia: Differential aspects. J Bras Patol Med Lab 48: 255-258.

50. Milman N (2011) Iron in pregnancy: How do we secure an appropriate iron status in the mother and child? Ann Nutr Metab 59: 50-54.
51. Portaria $\mathrm{n}^{\circ}$ 1247, de 10 de novembro de (2014) Brasília: Ministério da Saúde, Brasil.

52. U.S. Preventive Services Task Force (2013) Screening for Iron deficiency anemia-including iron supplementation for children and pregnant women. 